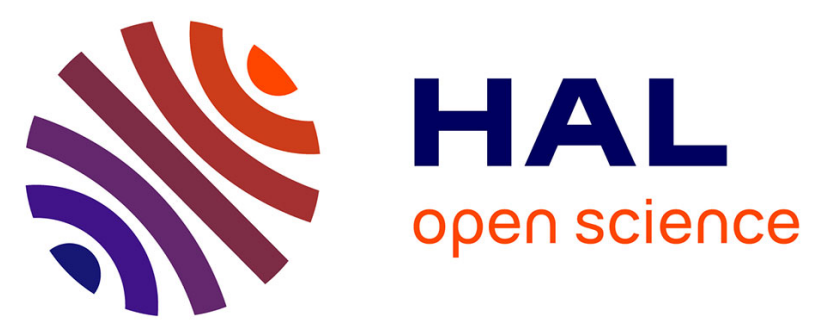

\title{
Representation of Interactions in a Multi-Level Multi-Agent Model for Cartography Constraint Solving
}

\author{
Adrien Maudet, Guillaume Touya, Cécile Duchêne, Sébastien Picault
}

\section{To cite this version:}

Adrien Maudet, Guillaume Touya, Cécile Duchêne, Sébastien Picault. Representation of Interactions in a Multi-Level Multi-Agent Model for Cartography Constraint Solving. 12th International Conference on Practical Applications of Agents and Multi-Agent Systems (PAAMS 2014), Jun 2014, Salamanca, Spain. pp.183-194, 10.1007/978-3-319-07551-8_16 . hal-01004369

\section{HAL Id: hal-01004369 https://hal.science/hal-01004369}

Submitted on 12 Jul 2017

HAL is a multi-disciplinary open access archive for the deposit and dissemination of scientific research documents, whether they are published or not. The documents may come from teaching and research institutions in France or abroad, or from public or private research centers.
L'archive ouverte pluridisciplinaire $\mathbf{H A L}$, est destinée au dépôt et à la diffusion de documents scientifiques de niveau recherche, publiés ou non, émanant des établissements d'enseignement et de recherche français ou étrangers, des laboratoires publics ou privés. 


\title{
Representation of Interactions in a Multi-Level Multi- Agent Model for Cartography Constraint Solving
}

\author{
Adrien Maudet ${ }^{1}$, Guillaume Touya ${ }^{1}$, Cécile Duchêne ${ }^{1}$, Sébastien Picault ${ }^{2}$ \\ ${ }^{1}$ Université Paris Est, IGN, laboratoire COGIT, Saint-Mandé, France \\ \{adrien.maudet, guillaume.touya, cecile.duchene\}eign.fr \\ ${ }^{2}$ LIFL, Université Lille 1, Villeneuve d'Ascq, France \\ sebastien.picaultelifl.fr
}

\begin{abstract}
The objective of cartographic generalisation is to simplify geographic data in order to create legible maps when scale decreases. It often requires to reason at different levels of abstraction (e.g. a building, a city). To automate this process, Multi-Agent approaches have been used for several years. Map objects (e.g. buildings) are modelled as autonomous entities that try to solve constraints through appropriate transformations. Yet, those approaches are not able to deal with all situations that appear between cartographic objects in a map. Indeed, though a map intrinsically involves objects that belong to several description, scale or organisation levels, there is no explicit multi-level representation in agent-based cartographic models. Thus we assume that the use of a multi-level multi-agent model would improve the automated generalisation process. Especially, the PADAWAN model is a multi-agent model offering multi-level capabilities which meet quite well the requirements for the multi-level organisation of cartographic objects. In this paper, we expose how we use this model on the one hand, to reify multi-level relations between cartographic agents, and on the other hand, to represent the constraints and the actions proposed to solve them, as interactions between the agents.
\end{abstract}

Keywords: Cartography, Cartographic Generalisation, Multi-Level Modelling, Spatialised Problems, Interactions Modelling, Constraints Solving.

\section{Introduction}

Map creation is a process with several stages aiming at drawing a legible map from geographic information. Today, geographic information is stored in databases where different objects (e.g. building, roads) are implemented with geometric shapes (e.g. point, polyline, polygon) and other non-geometric attributes (e.g. road name, building type). One characteristic of a map is its scale. Depending on the scale, information will not be shown, because room to display the same portion of the real world is smaller at a smaller scale. Among differences we may state various modifications, for instance: symbols may be changed (e.g. a building may be symbolised with its original shape, or a specific symbol depicting its function); the shape may be simplified (the detail of the line of a road may be more or less precise); some objects are not 
always displayed (i.e. small paths are not displayed on a road map, but they are displayed on a town map); some objects may be shown as a whole (i.e. close buildings in district centres are shown as a whole instead of being individually drawn).

All these modifications are motivated by the need to fit for the visual perception levels of the final user of the map. Yet, the information needs to remain semantically true (e.g. if the position of a building is allowed to change, it is not allowed to go to the other side of the road).
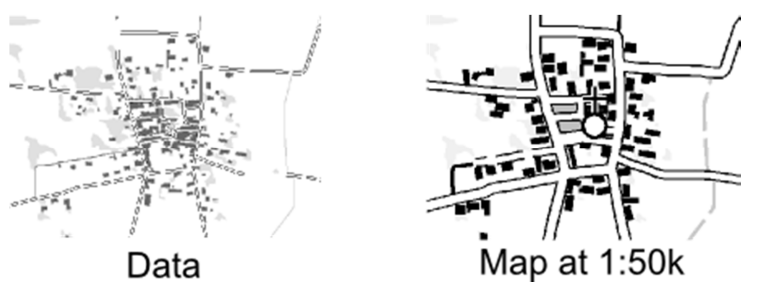

Fig. 1. Initial data generalised at the 1:50 000 scale.

This simplification process is called cartographic generalisation. Figure 1 shows an instance of generalisation. Its automation is a complex question. First, specific transformation algorithms have been designed in order to simplify the shape of objects, modify them and satisfy readability constraints (e.g. buildings must be above a minimum size, symbols have to be sufficiently spaced). But some algorithms involve several objects, and some objects are involved in more than one algorithm. The application of the appropriate algorithms to satisfy the constraints of objects of a map is a research question in geographic information science. Some solutions have been proposed. Among the different approaches, some are based on multi-agent models [1], [2], [5], [8], [11]. In multi-agent approaches for generalisation, geographical objects are modelled as agents trying to satisfy their own constraints. Some of these models handle the organisation in levels of geographical objects, because it has been shown that generalisation sometimes requires to reason on groups of objects considered as a whole [20] (e.g. a building is a part of an urban block, i.e. a portion of space bounded by roads). However, this organisation is rigid and the unsolved issues involve more complex relations between geographic objects, especially when they belong to several organisation or description levels [13]. Thus, we assume that using a more flexible multi-levels formalism may help us to tackle the unsolved issues. The purpose of this paper is to explain the whole approach, and develop the first steps of our ongoing work, consisting in defining a generic model to express multi-level interactions in an agent oriented approach for generalisation, and adapting behaviours of agents from existing models to the new one.

The paper is organised as follows. Related works on multi-agent systems for generalisation and on geographical multi-level organisation are presented in section 2 . Two opposite solutions (multi-model vs. improved multi-level approaches) are discussed in section 3; then section 4 addresses the issue of adapting one of the multilevel solutions to the existing multi-agent constraint-solving systems. Implementation and results are presented in section 5 , before we give some perspectives on this work. 


\section{Multi-Agent Approaches for Automated Generalisation}

In multi-agent generalisation models, the agents are either geographical entities (e.g. buildings, roads, towns) $[5,8,11,18]$, or the points that compose their geometry ([1, 11]. The purpose of these agents is to solve constraints that express some legibility conditions about geographical objects, as well as characteristics preservation conditions. Constraints affect either a single object (e.g. a minimum size constraint: a building must remain large enough to fit a perception threshold), or two objects (e.g. a proximity constraint: a road and a building must stay close to each other), or a group of objects (e.g. a density preservation constraint on an urban block involving all the buildings inside it).

In order to solve these constraints, geographic objects modelled as agents apply algorithms modifying their own geometry: the building grows bigger to satisfy its minimal size constraint; the building steps back from the road in order to satisfy the proximity constraints; the urban block eliminates some of its buildings in order to maintain its density while its buildings grow. The way the application of algorithms works depends on the different models. Among them, we are especially interested in AGENT, CartACom and GAEL, which are complementary to each other, and which all extend the model by [1]. This section briefly describes these three models, and then explains why the way relations are described may require a new perspective to go further in automatic generalisation quality.

\subsection{AGENT, CartACom and GAEL Multi-Agent Models for Generalisation}

AGENT ([2], [18], also formalised by [7]) is a model describing a hierarchical structure between agents: micro agents describe basic geographical entities (e.g. buildings, roads) and meso agents describe more complex entities composed by other agents, micro or meso (e.g. an urban block is composed of buildings). Meso agents share a hierarchical relation with their components. As explain in [19], this hierarchical relation implies different roles for a meso: coordinator, when the meso acts as a scheduler and activate its components; as a legislator, when the meso modifies its component and as a controller when the meso controls the result of the generalisation of its components. The behaviour of the agents follows a trial-and-error life cycle chaining constraints assessment, transformation, improvement evaluation, and commit or backtrack. It has an in-depth backtrack capability, resulting in an informed exploration of a states tree (described e.g. in [22]). AGENT proves effectiveness in urban zones, where the geographical entities are organised in hierarchies, and for the generalisation of roads, where a road may be subdivided into parts, which may generalise themselves, but need an agent to supervise the operations and maintain continuity between sections.

CartACom [3], [5] is a model managing transversal relations between agents. All agents interact in a same level. Here, constraints are shared by two agents and are called relational constraints. To satisfy them, an agent may either modify its own geometry, or try to modify the agent sharing the relation, which, due to the autonomy of the agents, requires to dialogue with it: ask, wait for an answer, and adapt its be- 
haviour to the response. The behaviour of the agents follows a life cycle similar to the one of AGENT but where only the last action can be backtracked. The scheduling of the agents is managed by a common scheduler, which uses some specific rules, like giving priority to an agent who just received a message. CartACom proves effectiveness in rural zones, where the density of objects is low.

The GAEL model [8] subdivides micro objects into primitives (points, segments, triangles) called submicro objects. Points of micro objects are modelled as agents trying to satisfy constraints to maintain the shape of related submicro objects. Micro objects are modelled as agents too, and share some constraints with other geographic agents like in CartACom. When a micro object is activated as a GAEL agent, it activates its point agents, and after acting, each point agent activates neighbouring points. GAEL proves effectiveness for the generalisation of background field objects (e.g. relief) consistently with foreground elements (e.g. rivers, roads, buildings).

When focusing on the multi-level aspect of these three models, different organisations may be noticed. In CartACom, all objects belong to the same level. AGENT suggests a tree-like structure, where components are part of meso objects, and modifications on these parts modify the meso agent itself. GAEL introduces interactions between two levels: micro and submicro, but in very specific ways. The ways levels and relations are modelled in these three models are very specific, and these models fail to handle more complex situations.

\subsection{Unsolved issues and new kinds of relations}

Those approaches of automated generalisation cover a lot of situations, but some cases are not well handled yet. We assume that agent oriented models may be used to handle these cases. But, as explained in the previous section, existing agent oriented models only enable to model specific kinds of interactions between geographical objects. The issues we want to solve involve different kind of multi-level relations. For instance, in figure 2, there are three recognisable levels: the micro objects level (buildings and roads), the urban blocks level, and between both, the aggregate level involving two adjacent buildings. The aggregate is, like the buildings, part of the urban blocks, and inside this urban block may need to interact as a whole with other buildings. But the adjacent buildings, although they are part of an aggregate, need to individually maintain their transversal relations with other buildings. Then, a solution to automatically generalise this situation requires:

- transversal relations between micro objects,

- hierarchical relations

- between micro objects and the urban block,

- between buildings and the aggregate,

- between the aggregate and the urban block,

- diagonal relations between the aggregate and the buildings outside this aggregate. 


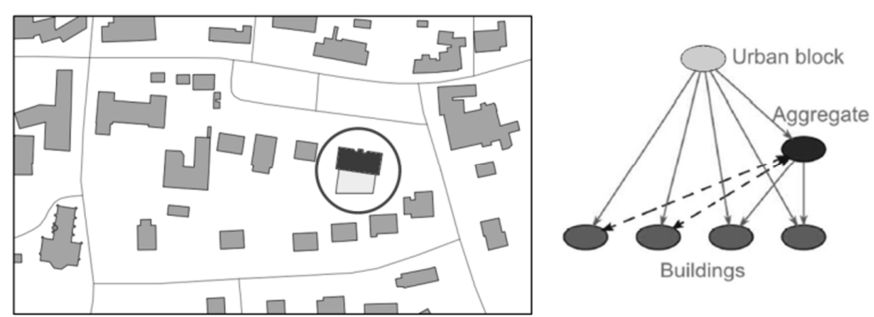

Fig. 2. Two adjacent buildings compose an aggregate. Aggregated buildings need to have normal interactions for buildings, but the aggregate also needs to interact as a whole.

In [13], other situations are exhibited. In particular, different kinds of hierarchical relations need to be modelled. An inclusion relation (i.e. a bus station on a road) [10] is different from a composition relation (i.e. a building is a part of an urban block). All these situations require a more flexible and more generic way to express relations and interactions between objects in different levels. This is why we propose to explore the use of a multi-level model.

\section{A Multi-Level Models to Better Handle Relations}

In this section, we expose our approach, consisting in using an appropriate existing multi-level model. Our objective is to get a model making explicit both hierarchical and transversal relations. First, we will explain why a multi-model approach is not suitable, then we will introduce the model we chose to base on while explaining why it was chosen.

\subsection{Multi-model solutions}

The goal of our work is not the mere fusion of existing models into a single one, but the design of a new one with more flexibility, in order to manage new kinds of interactions. These considerations are quite decisive in the choice of adaptations that have to be made to the existing models. Using a multi-model solution for generalisation was previously explored in [4] where three scenarios are introduced so as to improve multi-agent solution for generalisation. First, a multi-model method for generalisation was proposed, and developed in [24]. The purpose of this model is to identify areas, and apply the more appropriate process (a process may be an agent-oriented method or another kind of process, like a least square optimisation solution proposed by [9]). This solution provides good results, but is dependent on the processes capacity to handle all situations.

Second, a multi-agent multi-model system where agents may behave alternately as AGENT agents and CartACom agents was proposed. This kind of approaches raises several issues such as detecting the appropriate model in each situation, or ensuring the consistency of internal data used by a model when another one is used. Some methods are suggested in the simulation literature, like in [26]. But, the way to detect 
the need to change model is specific to each situation. Adding mechanisms for detecting model commutations seems very redundant with the existing constraints, which are already used as controller. We believe that generalisation requires an alternative solution. It may be to have all information in a same level, in order to choose in a single process which agent will be activated, and which algorithm will be apply. This implies to have a single model mimicking the behaviour of all three existing models.

\subsection{A multi-level multi-agent model: PADAWAN}

As exposed in [15], there has been a recent raise of the issue of multi-level modelling in multi-agent systems. Having analysed and compared existing multi-level multiagent models with regards to the needs in cartographic generalisation, including [6], [14], [21], [23], [25], our choice was to use the PADAWAN model [16], where the non-tree-like organisation of levels and the flexible definition of environment seem wide enough to model the complex relations between our geographical objects.

PADAWAN is a multi-level multi-agent model for simulation, allowing agents to get relations in several environments. It relies upon an interaction-oriented approach (IODA) [12]. The expression of agents' behaviour in the IODA formalism is based on interactions, that describe through a condition/action rule a behaviour that occurs between two entities. The entities involved in an interaction are a source, i.e. an agent, and a target, which may be one or several agents, or the source itself - for reflexive interactions. The behaviour of agents of a given environment is expressed using an interactions matrix. When an interaction between a family of sources and a family of targets is feasible, it is assigned to the intersection of the line of the source and the column of the target. This assignment links an interaction to a priority of execution and a maximum distance between the source and the target.

The description of an interaction consists in three distinct aspects: a trigger, preconditions and actions to do. The trigger expresses the motivation for the agent to do the interaction (e.g. in an ecosystem simulation "eat" may have "be hungry" as trigger). Preconditions express external conditions to satisfy ("get some food"). Both criteria need to be satisfied in order for an interaction to become feasible. Trigger, preconditions and actions are described using abstract primitives, which are to be concretely implemented by agents.

Compared with IODA, PADAWAN includes a multi-environment aspect. Agents may be situated inside one or more environments, and each environment may be encapsulated inside an agent, so that environments might be interlinked in a non-treelike way. Each environment gets its own interaction matrix, allowing the definition of behaviour rules specific to each environment. Added to the expression of relations between hosted agents, this matrix allows the expression of relations between a host and the hosted agent. 


\section{$4 \quad$ Adapting AGENT and CartACom}

In order to build the new model, we have to express the behaviour of original generalisation models within the PADAWAN paradigm, while gaining genericity. The adaptation of interactions from AGENT and CartACom models is the first step of our process seeking to solve remaining generalisation issues.

\subsection{Differences between simulation and problem solving}

While PADAWAN is designed for multi-agent simulation, map generalisation is a constraint-driven problem. In the first case, the objective is to simulate a phenomenon in order to make observations on a system. In a constraints solving problem, the objective is to obtain satisfying results with a minimum cost. Thus, the definition of the perception of the environment by an agent will not be motivated by the intention of realism (i.e. the range of the field of vision of an animal), but instead by care of efficiency: an agent will perceive all necessary things in order to be generalised at best. In both situations, the perception will be limited, but by differently defined criteria. Similar differences occur when considering life cycle and time aspects.

These differences are not, a priori, an issue to the adaptation of PADAWAN to the solving of cartographic generalisation problems. The perception of the environment in PADAWAN is implemented at the interaction matrix level. Regarding life cycle, the PADAWAN interactions selection process is used in a specific life cycle. This aspect is developed in the next sections.

\subsection{Inversion of the perspective}

In figure 3, the processing schemas of both AGENT and CartACom on one hand, and PADAWAN on the other hand, are displayed. The behaviour of agents in AGENT and CartACom is motivated by constraints. During its life cycle, an agent questions its constraint having priority to be solved, and this constraint returns a set of actions that the agent may try to execute. For instance, a building agent needs to satisfy its minimum size constraint. The agent then questions this constraint, and the constraint returns the following set of actions to try: \{ "Grow up", "Change geometry to a bigger rectangle", "Eliminate yourself"\}).

An action in a PADAWAN interaction can easily be taken from an AGENT/CartACom action. Rules defined in the constraints of AGENT/CartACom models are merged with PADAWAN preconditions. The notion of constraint satisfaction naturally brings around the use of unsatisfaction as an interaction trigger.

Regarding the choice, by the agent, of the next action (resp. interaction) to execute, the perspective between AGENT/CartACom and PADAWAN is reverted: in AGENT, the agent chooses the next constraint to satisfy, and then deduces the next action to try (the first one in the list of actions to try it returns); in PADAWAN, the agent chooses its interaction from the whole set of the feasible ones. In other words, the entry point is constraints in AGENT, but interactions in PADAWAN. The switch is non-trivial, because in AGENT an action may be proposed by several constraints, 
and an action proposed by a constraint may be discouraged by another one. The first step of the adaptation of the action from AGENT and CartACom is to establish a way to express the opinion that a constraint may have on the realisation of an action.

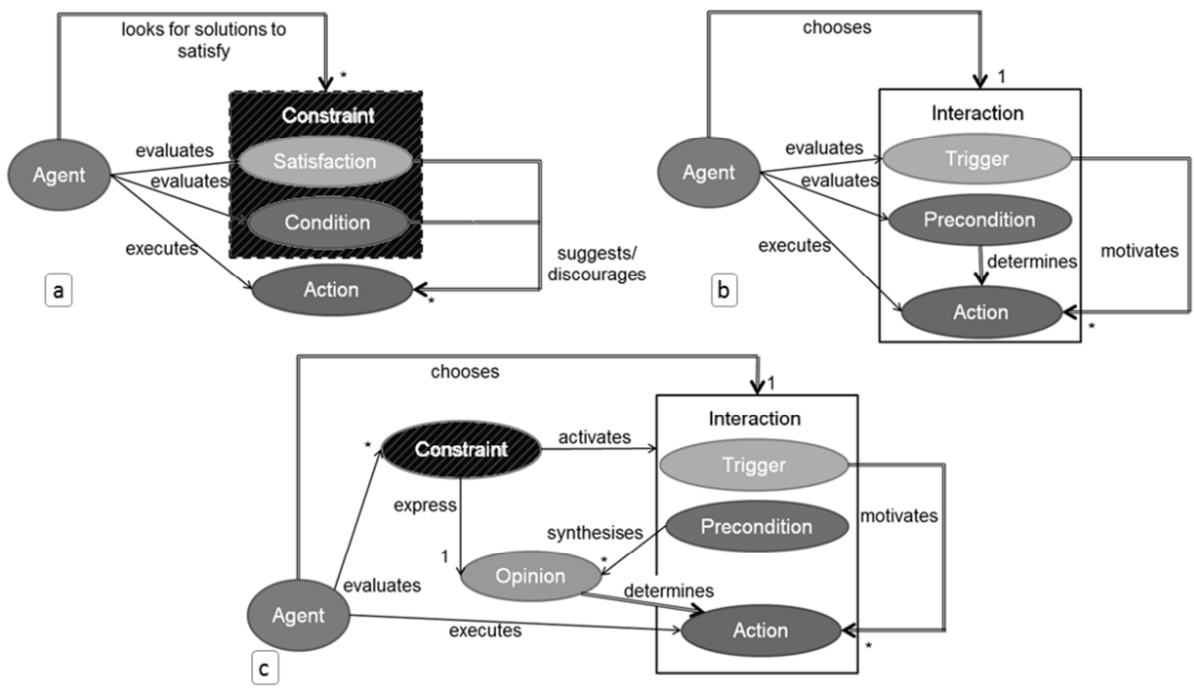

Fig. 3. (a) exposes the mechanism of AGENT and CartACom, (b) exposes the mechanism of PADAWAN, (c) is our proposal to use specific aspects of generalisation models in the PADAWAN models.

\subsection{Behaviour of the agents}

In existing generalisation models, a constraint may take a stand for (or against) an action in four different ways. In order to formalise this report, we introduce the notion of advices of a constraint. The four possible advices that a constraint may express are:

- indifferent, the default one, when there is no prior generalisation knowledge about the influence of the execution of an action on the satisfaction of this constraint,

- favourable, only when the constraint is unsatisfied, and generalisation knowledge assumes a positive influence of the action execution on the constraint satisfaction,

- unfavourable, opposite of the previous one, when the execution of the action is $a$ priori negative for the constraint,

- opposite, when the impact of the execution of the action will certainly be damaging, and may cause irreparable mistakes.

For instance, for a building, the unsatisfied "Squareness" constraint (expressing the willingness to replace almost right angles by strictly right angles) will be favourable to the "Simplify in Rectangle" action, but the "Preservation of the concavity" constraint (for objects with a concave shape) will be unfavourable to this action. Synthesising advices of constraints, an interaction may be a priori: 
- feasible, if at least one constraint is favourable, and the others are in the worst case indifferent;

- feasible, but risky, if at least one constraint is favourable, at least one is unfavourable, and none is opposite;

- unfeasible, when at least one is opposite, or when there is no favourable constraints.

The precondition notion of PADAWAN is substituted by this advice model. This allows a first selection of feasible interactions, but it is not sufficient to choose the best interaction. In the AGENT and CartACom models, the constraint having priority is selected according to following factors, in order of decreasing importance: the importance of the constraint (a constraint will not be relaxed before another one with a lower importance), the current unsatisfaction, and a weight factor given by the constraint for the action. At the moment, we use here a basic heuristic that needs to be enhanced. For the adaptation of PADAWAN, we use the notion of trigger to organise interactions.

The constraint aspect of AGENT, CartACom and GAEL was translated into the PADAWAN paradigm using this expressed advice model. Interactions were defined and included in interaction matrices sets, specific to some concrete experiments describes in section 5 . We defined new life cycles for the agent based on these matrices. These life cycles are based on the PADAWAN life cycle, but add adaptations specific to our generalisation problem. One of the adaptations is the fact that the life cycle of an agent in generalisation allows to revert a modification, if a modification is assessed as having a negative effect on constraints satisfaction. So, when the original PADAWAN life cycle for simulation chooses one action sequence, in our model for generalisation, the agent identifies a ranked list of possible actions, and tries and tests the first one. If one of them gives a perfect solution (all constraints are satisfied), the life cycle is over. If the impact is positive, but not perfect, the model proposes another list of realisable interactions, computed from the new result, and tries another one. If the impact is negative, the interaction is cancelled, and the next one in the list is tried. Two life cycles have been implemented with simple and extended backtracking respectively, corresponding to the original CartACom and AGENT life-cycles.

\section{$5 \quad$ Implementation and results}

Our first experiment was to try to reproduce the existing models results with the PADAWAN paradigm. To do that, we used the matrices shown on figure 4a for AGENT and on figure $4 \mathrm{~b}$ for CartACom. As we can see, no interaction between host agent and hosted agents may be seen in the CartACom matrix, and no action between two hosted elements may be seen in the AGENT matrix which translates the fact that interactions between agents are hierarchical in AGENT and transversal in CartACom. We used the life-cycles with simple and extended backtracking for CartACom and AGENT respectively. As a result, we managed to reproduce the results of the original models, in term of effectiveness (the quality of the results is the same), and in term of efficiency (the number of time-consuming operations is the same). 


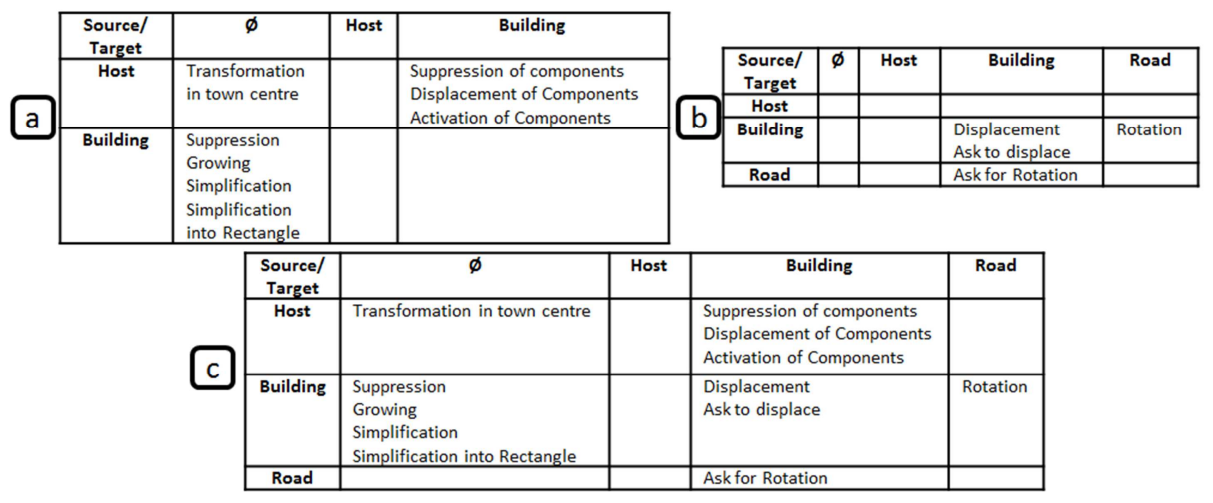

Fig. 4. Different matrices for our experiments. (a) matrix for urban an urban block in AGENT, (b) matrix for the map environment in CartACom, (c) matrix for urban block in our combined experiment.

For our second experiment, we tried to mix agent behaviours stemming from both AGENT and CartACom models, on a simple application case (to begin) that however is a remaining problem of generalisation. When generalising urban areas with buildings to a scale of $1: 25 \mathrm{k}$ or smaller, we want to ensure that buildings almost parallel to a road change to strictly parallel. Indeed, the exact relative orientation of a building is, for this kind of scale, information surplus. Applying the rotation operation, based on a relational constraint of parallelism, is already done in rural areas using CartACom. We want here to introduce this transformation also when generalising an urban area with the AGENT paradigm. We use the matrix given in figure $4 \mathrm{c}$, and the life cycle with simple backtracking. The main difficulty was to calibrate the parameters controlling the order in which the actions are tried. This calibration was done empirically, with progressive refinement. Despite this methodology not being satisfactory, we managed to get some satisfying results, as shown in figure 5 . This proves that we may add new interactions in a simple way, and opens perspectives for solving more complex problems using interactions that are not purely hierarchical or transversal.
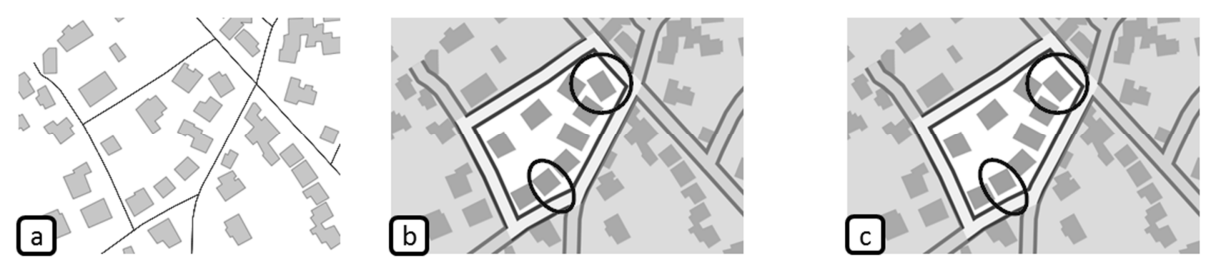

Fig. 5. (a) non-generalised data, (b) generalised data in 1:25k, using AGENT (only the central block was generalised), (c) generalised data using our PADAWAN modelling with buildings aligned to the road. 


\section{Conclusion and perspectives}

In order to solve remaining generalisation issues, we showed that modelling interactions of agents using a multi-level formalism is quite helpful. Therefore, we used a multi-level simulation model and proposed an adaptation for the specificities of a constraints driven problem. As a first step, we manage to reproduce the results of existing models, and to solve some relatively simple issues handling multi-level relations, like the orientation of building in urban area (Figure 5), or the generalisation of dead-ends and of their neighbourhood [13].

In this paper, we presented the first steps of a work in progress with imperfect results demanding manual corrections. Our next step will be to propose a generic way to orchestrate possible interactions and to calibrate parameters used to order possible interactions. Another point is to propose a better heuristic for selecting interactions. Then, we will propose a solution to integrate the GAEL agents behaviour in our system, focusing on topographic maps with trekking and tourism data upon.

\section{References}

1. Baeijs C., Demazeau Y. and Alvares L.: SIGMA: Application of Multi-Agent Systems to Cartographic Generalization. In: MAAMAW, volume 1038 of Lecture Notes in Computer Science, page 163-176. Springer, (1996)

2. Barrault M., Regnauld N., Duchêne C., Haire K., Baeijs C., Demazeau Y., Hardy P., Mackaness W., Ruas A. and Weibel R.: Integrating Multi-agent, Object-oriented, And Algorithmic Techniques For Improved Automated Map Generalization. In: 20th International Cartographic Conference, vol.3, 2110-2116 (2001).

3. Duchêne C.: Généralisation cartographique par agents communicants : le modèle CartACom. PhD Thesis, Université Paris 6 - Pierre et Marie Curie, Paris, France (2004).

4. Duchêne C. and Gaffuri J.: Combining three multi-agent based generalisation models: AGENT, CartACom and GAEL. In: 13th International Symposium on Spatial Data Handling, 277-296 (2008)

5. Duchêne C., Ruas A. and Cambier C.: The CartACom model: transforming cartographic features into communicating agents for cartographic generalisation. International Journal of Geographic Information Science, 26(9):1533-1562 (2012)

6. Ferber J., Michel F. and Baez J.: AGRE: Integrating environments with organizations. In: Environments for Multi-Agent Systems, vol. 3374 of Lecture Notes in Computer Science, chap. 2, 48-56-56 (2005)

7. Fernandes K. : Systèmes Multi-Agents Hybrides : Une approche pour la Conception des Systèmes Complexes. PhD Thesis, Université Joseph Fourier, Grenoble, France (2001)

8. Gaffuri J., Duchêne C. and Ruas A.: Object-field relationships modelling in an agent-based generalisation model. In: Proceedings of 11th ICA workshop on Generalisation and Multiple Representation, Montpellier, France (2008)

9. Harrie L. and Sarjakoski T.: Simultaneous graphic generalization of vector data sets. In: Geoinformatica 6 (3), 221-233 (2002)

10. Jaara K., Duchêne C. and Ruas A.: A model for preserving the consistency between topographic and thematic layers throughout data migration. In: 15th International Symposium on Spatial Data Handling (SDH'12), Bonn, Germany (2012) 
11. Jabeur N., Boulekrouche B. and Moulin B.: Using multi-systems to improve real time mobile map generation. In Lamontagne and M. Marchand (Edts), Advances in Aritifical Intelligence, Springer Verlag LNAI 4013, 37-48 (2006)

12. Kubera Y., Mathieu P. and Picault S.: IODA: an interaction-oriented approach for multiagent based simulations, in: Journal of Autonomous Agents and Multi-Agent Systems (JAAMAS), vol. 23 n. 3, p. 303-343. Springer (2011)

13. Maudet A., Touya G., Duchêne C. and Picault S.: Improving multi-level interactions modelling in a multi-agent generalisation model: first thoughts. In: Proceedings of 16th ICA workshop on Generalisation and Multiple Representation, Dresden, Germany (2013)

14. Minar N., Burkhart R., Langton C. and Askenazi M.: The Swarm simulation system: A toolkit for building Multi-Agent simulations. Technical Report 96-06-042, Santa Fe Institute (1996)

15. Morvan G.: Multi-level agent-based modelling - A literature survey. http://arxiv.org/abs/1205.0561

16. Picault S. and Mathieu P.: An Interaction-Oriented Model for Multi-Scale Simulation. In: 22nd International Joint Conference on Artificial Intelligence (IJCAI/AAAI), Barcelona, Spain, 332-337 (2011)

17. Renard J., Gaffuri J. and Duchêne C.: Capitalisation problem in research -example of a new platform for generalisation: CartAGen. In: 12th ICA Workshop on Generalisation and Multiple Representation, Zürich, Switzerland (2010)

18. Ruas A.: Modèle de généralisation de données géographiques à base de contraintes et d'autonomie. PhD Thesis, Université Marne La Vallée (1999)

19. Ruas A.: The Roles of Meso Objects for Generalisation. In: 9th International Symposium on Spatial Data Handling, Beijing, China, 3b, 50-63 (2000)

20. Ruas A. and Plazanet C.: Strategies for automated map generalisation. In: 7th International Symposium on Spatial Data Handling (SDH'96), Delft, Netherlands, 319-336 (1996)

21. Soyez J.B., Morvan G., Dupont D. and Merzouki R.: A Methodology to Engineer and Validate Dynamic Multi-level Multi-agent Based Simulation. In: Multi-Agent Based Simulation XIII, volume 7838 of Lecture Notes in Artificial Intelligence, 130-142 (2013).

22. Taillandier, P. and Gaffuri, J.: Improving map generalisation with new pruning heuristics. In: International Journal of Geographical Information Science, Taylor \& Francis, 26, 1309-1323 (2012).

23. Taillandier P., Vo D.A., Amouroux E. and Drogoul A.: GAMA: A simulation platform that integrates geographical information data, agent-based modeling and multi-scale control. In: Principles and Practice of Multi-Agent Systems, volume 7057 of Lecture Notes in computer Science, 242-258 (2012).

24. Touya G. and Duchêne C.: CollaGen: Collaboration between automatic cartographic generalisation processes. In: Advances in Cartography and GIScience, 541-558 (2011)

25. Vo D.A., Drogoul A. and Zucker J.D.: An operational Meta-Model for handling multiple scales in Agent-Based simulations. In: International Conference on Computing \& Communication Technologies, Research, Innovation, and Vision for the Future (2012)

26. Yilmaz L., Lim A., Bowen S. and Ören T.: Requirements and Designs Principles for Multisimulation with Multiresolutions, Multistage and Multimodels. In: Proceedings of the 2007 Winter Simulation Conference (2007) 